\title{
Journée des malades 2014
}

La Suisse célèbre la «Journée des malades» qui se déroule tous les ans le premier dimanche du mois de mars, soit cette année, le 2 mars 2014. Cette édition qui marque les 75 ans d'existence de l'association éponyme est consacrée au thème suivant: «Charges psychiques: le courage d'en parler!». Un débat dans lequel les médecins ont un rôle à jouer.

Ursula Steiner-König

Déléguée de la FMH au Comité central de la Journée des malades

\begin{abstract}
Que ce soit sous l'angle du TDAH, de l'autisme et du syndrome d'Asperger, de l'épuisement professionnel ou de la dépression, le thème des charges psychiques revient régulièrement dans les médias. Pourtant, la stigmatisation des personnes confrontées à des difficultés psychiques voire à une maladie ne diminue pas mais aurait plutôt tendance à s'accroître: une réalité nourrie par le monde politique avec des conséquences que les médecins comme les patients doivent aujourd'hui affronter. Il est extrêmement préoccupant de voir que le canton de Berne par exemple adopte des mesures d'économie qui amènent des malades psychiques sans autre solution de placement à devoir «simplement» quitter l'hôpital dès lors qu'une prise en charge hospitalière ne semble plus indiquée. Malheureusement, il est avéré que bien trop peu d'institutions sont en mesure de recevoir et de prendre en charge ces patients.
\end{abstract}

\section{Penser aux malades, leur rendre visite, ne pas les laisser de côté lorsqu'ils sont atteints de maladies chroniques.}

Du point de vue médical, la fermeture du Département de psychosomatique/Lory-Haus à la Clinique universitaire de l'Hôpital de l'Ile à Berne est, elle aussi, peu compréhensible. En effet, c'est non seulement la seule unité universitaire permettant la prise en charge hospitalière de patients atteints de troubles psychosomatiques, mais en plus j'ai l'impression que les politiciens en charge de la santé oublient que malgré des diagnostics (qui, selon eux, ne sont pas dignes de figurer dans le catalogue des maladies traitées par une clinique universitaire), des personnes souffrent de leur pathologie. La souffrance ne semble pas avoir sa place dans le monde de l'économie et de la rationalisation.

De même, une situation qui s'est récemment pro-

Correspondance:

Dr Ursula Steiner-König Beim Goldenen Löwen 3 CH-4052 Bâle

usteiner[at]hin.ch qu'il n'était pas en mesure de suivre toute la procédure administrative. Ce dernier souffre effectivement d'une forme sévère de schizophrénie et il n'appréhende pas la réalité comme tout un chacun. Même les recours de la fratrie n'ont manifestement pas réussi à émouvoir les responsables politiques. «Un être qui n'est pas normal ne peut donc pas être naturalisé», ont-ils visiblement pensé. Evoquons enfin tous les patients présentant des troubles douloureux somatoformes dont font état pratiquement toutes les disciplines médicales et que l'assurance-invalidité refuse de reconnaître comme malades susceptibles d'obtenir des prestations AI ou une rente. Les médecins chargés des expertises ont tendance à présumer davantage de capacités à ces patients que ne le feraient les médecins traitants qui les accompagnent sur de longues périodes. Ainsi, il n'est pas rare que le médecin traitant évalue différemment l'incapacité d'exercer une activité professionnelle que le médecin-conseil dont la mission consiste à exprimer un avis sur la base d'un examen ponctuel tout en gardant présent à l'esprit le fait d'accorder le moins possible de rentes ou de prestations de l'AI. Une situation peu enviable entre le marteau et l'enclume.

Par ailleurs, n'oublions pas que le nombre de candidats à la spécialisation en psychiatrie et psychothérapie a énormément baissé et que de ce fait, ces prochaines années, de nombreux cabinets de psychiatres et de psychothérapeutes ne trouveront pas de successeur suisse. Dans ce contexte, tous les médecins sont aujourd'hui appelés à faire preuve de courage et d'ouverture au dialogue, et à aborder avec leurs patients la question d'éventuelles charges psychiques; ne seraitce que pour évoquer des possibilités de traitements différents.

Par chance, un nombre important de collègues étrangers accomplissent aujourd'hui leur formation postgraduée en psychiatrie et psychothérapie selon le cursus suisse qui, du point de vue qualitatif, est de loin meilleur que d'autres formations étrangères similaires reconnues dans l'UE. Nous savons aussi que, ces prochaines années, de nombreux psychothérapeutes spécialisés formés en psychologie vont venir élargir le marché. Il ne s'agit pas de débattre de leur aptitude à comprendre les phénomènes psychosomatiques 
mais de souligner que la relation de confiance entre le patient et le médecin est beaucoup plus déterminante que la théorie et le savoir d'une personne spécialisée.

Bien évidemment, l'appréciation des charges psychiques n'exclut pas les connaissances spécialisées mais celles-ci n'ont pas le don d'ubiquité. Dès lors, il faut pouvoir compter sur une bonne collaboration interdisciplinaire, que mes expériences en tant que professionnelle «du terrain» m'ont heureusement permis de connaître; même si certains préféraient parfois préserver leur pré carré. A propos de «courage d'en parler», sans répéter ce qui figure sur la fiche d'information de la «Journée des malades» (www.journeedes malades.ch), j'aimerais simplement ajouter que nous médecins devons être prêts au dialogue interdisciplinaire, bénéfique non seulement pour nous mais surtout pour nos patients, sans pour autant engendrer de coûts supplémentaires.

\section{Les $\mathbf{7 5}$ ans de la Journée des malades}

L'importance d'avoir une pensée toute particulière pour les malades, de leur rendre visite, de ne pas les laisser de côté lorsqu'ils sont atteints de maladies chroniques, tout cela ne devrait pas être limité au $1^{\text {er }}$ dimanche de mars mais nous habiter toute l'année. En créant la «Journée des malades», le Docteur Marthe Nicati, qui a durant de nombreuses années été au chevet des tuberculeux à Leysin, avait fixé les objectifs suivants:

- promouvoir de bonnes relations entre malades et bien-portants;
- rappeler aux bien-portants leur devoir vis-vis des personnes malades;

- favoriser la compréhension pour les besoins des malades;

- reconnaître l'engagement de toutes celles et ceux qui à titre professionnel ou privé s'engagent pour les patients.

Mme Nicati avait compris que les patients en sanatorium souffraient de solitude, ce à quoi elle a consacré son action. Aujourd'hui, même en dépit des progrès de la médecine, ses idées n'ont rien perdu de leur pertinence. Le Comité central de la «Journée des malades» se félicite de voir que son institution perdure depuis trois quarts de siècle et lui souhaite de tout cœur encore de belles années.

\section{Une carte et des affiches}

A l'occasion de cet anniversaire, ANNA a dessiné pour nous une petite carte comportant des souhaits de rétablissement au verso: une petite attention qui peut être utilisée tout au long de l'année. Sur le site de la «Journée des malades» www.journeedesmalades.ch, nos collègues trouveront en outre des affiches à télécharger ainsi que des cartes en trois langues à consulter et le cas échéant à commander.

Une affiche, signe de notre solidarité, a certainement sa place dans les salles d'attente.

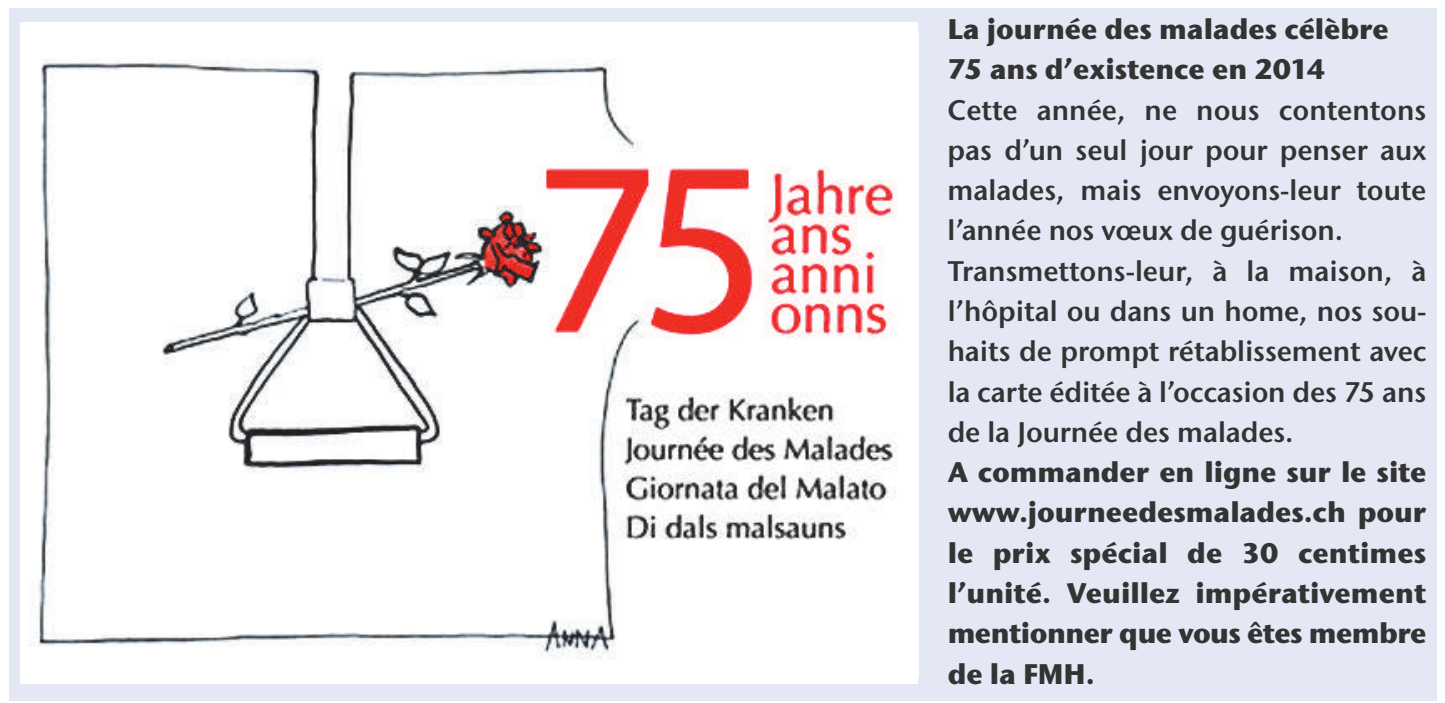

